\title{
Wirksamkeit und Sicherheit des humanen monoklonalen Interleukin-12/23- Antikörpers Ustekinumab bei Patienten mit Psoriasis: Ergebnisse der randomisierten, doppel-blinden, plazebokontrollierten Phase-III-Studien PHOENIX 1 und PHOENIX 2
}

\author{
Efficacy and Safety of Ustekinumab, a Human Monoclonal Interleukin-12/-23 Monoclonal Antibody, in Patients \\ with Psoriasis: Results from the Randomised, Double-Blind, Placebo-Controlled Phase-III-trials PHOENIX 1 and \\ PHOENIX 2
}
Autor
K. Reich
Institut
Dermatologikum Hamburg

\section{Bibliografie}

DOI $10.1055 / \mathrm{s}-0028-1103404$

Akt Dermatol 2009; 35:

29-34 ๑ Georg Thieme

Verlag KG Stuttgart · New York ISSN 0340-2541

Korrespondenzadresse

Prof. Dr. med. Kristian Reich Dermatologikum Hamburg Stephansplatz 5 20354 Hamburg reich@dermatologikum.de

\section{Zusammenfassung \\ $\nabla$}

Interleukin-12 und Interleukin-23 spielen eine wichtige Rolle in der Pathophysiologie der Psoriasis. Der humane monoklonale Antikörper Ustekinumab bindet an die in beiden Zytokinen vorkommende Untereinheit p40 und blockiert auf diese Weise die Bindung der Interleukine an ihren Zielrezeptor. In zwei randomisierten, doppel-blinden, plazebokontrollierten Phase-III-Studien, PHOENIX 1 und PHOENIX 2, wurde die Wirksamkeit und Sicherheit von Ustekinumab als subkutane Injektionen an knapp 2000 Patienten mit mittelschwerer und schwerer Psoriasis untersucht $[1,2]$. Beide Studien hatten eine plazebokontrollierte erste Phase, in der $45 \mathrm{mg}$ oder $90 \mathrm{mg}$ Ustekinumab oder Plazebo jeweils an Woche 0 und 4 injiziert wurden. Nach 12 Wochen zeigte sich bei $67 \%$ bzw. $72 \%$ der mit Ustekinumab behandelten Patienten eine mindestens $75 \%$ ige Verbesserung des „Psoriasis Area and Severity Index“ (PASI 75 ) gegenüber $4 \%$ bei mit

\section{Einfiihrung}

$\nabla$

Psoriasis ist die häufigste immuninduzierte entzündliche Erkrankung der Haut $[3,4]$ : Weltweit sind schätzungsweise $2-3 \%$ der Bevölkerung betroffen $[5,6]$. Die chronische Psoriasis beeinträchtigt die Patienten deutlich in ihren physischen und psychischen Funktionen sowie in ihrer Lebensqualität [7]. Mit zunehmender Kenntnis der grundlegenden immunpathophysiologischen Veränderungen der Psoriasis gelingt es, Substanzen zu entwickeln, die mit hoher Selektivität in die veränderte Immunantwort eingreifen. Zu diesen Substanzen, den so genannten Biologika, gehören beispielsweise Antikörper oder Fusionsproteine, die gegen Oberflächenmoleküle auf T-Zellen oder den Tumornekrosefaktor $\alpha$ gerichtet sind [8].
Plazebo behandelten Patienten. Die Abnahme der Hautsymptome war begleitet von einer deutlichen Verbesserung der Lebensqualität. Nach 12 Wochen berichteten $55 \%$ bzw. $71 \%$ der mit $45 \mathrm{mg}$ bzw. $90 \mathrm{mg}$ behandelten Patienten (Plazebo: 4\%), nicht durch Psoriasis beeinträchtigt zu sein (Dermatology Life Quality Index '0' oder '1'). Die bisher über 76 Wochen vorliegenden Daten zeigen eine konstante Wirksamkeit von Ustekinumab bei Fortführung der Therapie mit Injektionen alle 12 Wochen. Ustekinumab zeigte in der Kurzzeit- und Langzeittherapie eine gute Sicherheit und Verträglichkeit. Unerwünschte Ereignisse, schwerwiegende unerwünschte Ereignisse oder unerwünschte Ereignisse, die zu einem Studienabbruch führten, traten bei mit Ustekinumab behandelten Patienten nicht häufiger als bei mit Plazebo behandelten Patienten auf. Damit erweist sich Ustekinumab als eine neue wirksame und sichere Therapieoption für die Behandlung von Patienten mit mittelschwerer und schwerer Psoriasis.

Der noch nicht zugelassene humane monoklonale Interleukin-12/-23-Antikörper Ustekinumab (CNTO 1275; Centocor Inc. Malver, PA, USA) verfügt über ein neues Wirkprinzip: Das Biologikum richtet sich gegen die beiden Interleukine (IL) 12 und 23, die in der Pathophysiologie der Psoriasis eine bedeutende Rolle spielen. IL-12 und IL-23 sind Heterodimere, die beide die Untereinheit p40 enthalten. Genetische Polymorphismen der in Psoriasis-Plaques überexprimierten Untereinheit p40 [9] als auch des Rezeptors für IL-23 wurden mit Psoriasis in Zusammenhang gebracht [10]. Präklinische Studien sowie erste klinische Studien deuten auf eine Rolle der p40-haltigen Zytokine in der Pathogenese der Erkrankung hin [11]. Ustekinumab bindet mit hoher Affinität und Spezifität an die p40-Untereinheit von IL-12 und IL-23 und verhindert auf diese Weise die 
Bindung der beiden Interleukine an ihren Zelloberflächen-Rezeptor IL12R $\beta 1$.

Um das therapeutische Potenzial des neuen monoklonalen Antikörpers zu bestätigen, wurde in den beiden Phase-III-Studien PHOENIX 1 und PHOENIX 2 die Wirksamkeit und Sicherheit von Ustekinumab in zwei verschiedenen subkutanen Dosierungen bei Patienten mit mittelschwerer bis schwerer Psoriasis untersucht.

\section{Patienten und Methoden \\ $\nabla$}

In den beiden randomisierten, doppel-blinden, plazebokontrollierten Studien nahmen knapp 2000 Patienten mit mittelschwerer und schwerer Psoriasis teil: In die Studie konnten Patienten eingeschlossen werden, die mindestens 18 Jahre alt waren, seit mindestens 6 Monaten an einer Plaque-Psoriasis litten, die mindestens $10 \%$ der Körperoberfläche einnahm, zu Studienbeginn einen PASI-Score von mindestens 12 aufwiesen und Kandidaten für eine Phototherapie oder systemische Therapie waren.

Das Design der beiden dreiarmigen Studien bestand jeweils aus drei verschiedenen Phasen.

In PHOENIX 1 wurden 766 Patienten für die erste Phase (Woche 0-12) und zweite Phase (Woche 12-40) drei verschiedenen Behandlungsarmen zugeordnet:

- 255 Patienten erhielten Ustekinumab $45 \mathrm{mg}$ in den Wochen 0 und 4 und danach alle 12 Wochen bis Woche 40 ,

- 256 Patienten erhielten Ustekinumab $90 \mathrm{mg}$ in den Wochen 0 und 4 und danach alle 12 Wochen bis Woche 40 und

- 255 Patienten erhielten Plazebo in den Wochen 0 und 4 mit anschließendem Cross-over auf 45 mg oder 90 mg Ustekinumab ab Woche 12 mit Injektionen in den Wochen 12, 16 und danach alle 12 Wochen bis Woche 40 .

Ab Woche 40 erfolgte dann die dritte Phase, in der die Patienten bis Woche 76 randomisiert eine Erhaltungstherapie erhielten oder die Behandlung unterbrochen wurde:

- Patienten, die seit Woche 0 mit Verum behandelt wurden und an Woche 28 und 40 eine PASI-75-Antwort aufwiesen (mindestens 75\%ige Besserung des PASI), wurden entweder weiter mit der jeweiligen Dosis Ustekinumab als Erhaltungstherapie behandelt oder erhielten so lange Plazebo, bis sie $50 \%$ ihrer PASI-Verbesserung verloren; dann wurde die vorherige Dosierung von Ustekinumab wieder angesetzt.

- Bei Patienten, die zu Beginn der Studie zuerst Plazebo erhielten, wurde die Behandlung ebenfalls abgesetzt und wieder angesetzt, wenn $50 \%$ ihrer PASI-Verbesserung verloren ging. Patienten, die zu Woche 28 oder Woche 40 einen PASI unter 50 (Non-Responder) hatten, wurden von der Studie ausgeschlossen. Teilresponder mit einem PASI 50 bis $<75$ zu Woche 28 oder 40 erhielten ihre Dosis alle 8 Wochen.

In PHOENIX 2 wurden 1230 Patienten ebenfalls für die Phase 1 (Woche 0-12) und Phase 2 (Woche 12-28) drei verschiedenen Behandlungsarmen zugeordnet:

- 409 Patienten erhielten Ustekinumab $45 \mathrm{mg}$ in den Wochen 0 und 4 und danach alle 12 Wochen bis Woche 28 ,

- 411 Patienten erhielten $90 \mathrm{mg}$ Ustekinumab in den Wochen 0 und 4 und danach alle 12 Wochen bis Woche 28 und

- 410 Patienten erhielten Plazebo in den Wochen 0 und 4 mit anschließendem Cross-over auf Ustekinumab ab Woche 12 mit Ustekinumab-Injektionen in den Wochen 12, 16 und danach alle 12 Wochen bis Woche 28 .
Ab Woche 28 erfolgte dann in der dritten Phase randomisiert die Weiterbehandlung alle 12 oder 8 Wochen je nach Verbesserung des PASI bis Woche 52:

- Patienten mit PASI-75-Antwort erhielten die jeweilige Dosierung Ustekinumab weiter alle 12 Wochen.

- Patienten mit partiellem Ansprechen, definiert als PASI $\geq 50$ und $<75$, erhielten ihre jeweilige Ustekinumab-Dosis randomisiert entweder weiterhin alle 12 Wochen oder als Dosisoptimierung alle 8 Wochen.

Patienten, die zu Woche 28 einen PASI unter 50 hatten, wurden von der Studie ausgeschlossen.

Beide Studien laufen in einer offenen Verlängerungsphase über einen Zeitraum von 5 Jahren weiter.

Der primäre Endpunkt in beiden Studien war der Anteil der Patienten, die an Woche 12 einen PASI 75 erreichten. Die wichtigsten sekundären Endpunkte waren die Verbesserung der Lebensqualität an Woche 12 gemessen anhand des „Dermatology Life Quality Index“(DLQI; Bereich 0-30 mit 0 und 1 = Lebensqualität nicht beeinträchtigt, 2 bis 5 Lebensqualität gering beeinträchtigt, 6 bis 10 Lebensqualität mäßig beeinträchtig, 11 - 20 Lebensqualität sehr stark beeinträchtigt, 21 - 30 Lebensqualität extrem stark beeinträchtigt) sowie eine globale Einschätzung des Arztes („Physicians' Global Assessment“ (PGA) mit 0 = erscheinungsfrei, 1 =minimal,$\quad 2$ = leicht,$\quad 3$ = mittelschwer,$\quad 4$ = ausgeprägt $5=$ schwer) von 0 oder 1 . Weitere sekundäre Endpunkte waren in PHOENIX 1 die Zeit ab Unterbrechung der Therapie mit Ustekinumab bei Patienten mit PASI-75-Antwort bis zum Wiederansetzen der Behandlung aufgrund des Abfalls der PASI-Verbesserung um 50\% sowie in PHOENIX 2 die Anzahl der Teil-Responder, die ab Woche 28 ihre Studienmedikation alle 8 Wochen erhielten und in Woche 40-52 wieder einen PASI 75 erreichten im Vergleich zu den Patienten, die ihre Studienmedikation durchgehend alle 12 Wochen erhielten.

\section{Ergebnisse}

$\nabla$

Die wesentlichen Patientencharakteristika waren in beiden Studien und auch in den jeweiligen Behandlungsgruppen vergleich$\operatorname{bar}(\bullet$ Tab. 1$)$.

Das mittlere Alter lag bei 46 Jahren, zwei Drittel der Studienteilnehmer waren Männer und das mittlere Körpergewicht betrug $92 \mathrm{~kg}$. Die meisten Patienten hatten eine lange bestehende

Tab. 1 PHOENIX 1 und PHOENIX 2. Vergleichbare demografische Ausgangsdaten und Krankheitsschweregrade.

\begin{tabular}{lll} 
& $\begin{array}{l}\text { PHOENIX 1 - } \\
\text { Gesamt [21] }\end{array}$ & $\begin{array}{l}\text { PHOENIX 2 - } \\
\text { Gesamt [22] }\end{array}$ \\
\hline randomisierte Patienten & 766 & 1230 \\
\hline männlich, $\mathrm{n}(\%)$ & $531(69,3 \%)$ & $840(68,3 \%)$ \\
\hline Alter (Jahre) $\dagger$ & $45,3(45,5)$ & $46,2(47,0)$ \\
\hline Gewicht (kg) $\dagger$ & $93,88(91,6)$ & $90,99(88,6)$ \\
\hline Dauer der Psoriasis (Jahre) $\dagger$ & $19,9(18,3)$ & $20,1(18,5)$ \\
\hline BSA (\%) $\dagger$ & $26,7(21,0)$ & $26,4(20,0)$ \\
\hline PsA, n (\%) & $258(33,7 \%)$ & $305(24,8 \%)$ \\
\hline PASI-Score (0-72) $\dagger$ & $20,22(17,6)$ & $19,61(17,5)$ \\
\hline PGA deutlich oder schwer, n (\%) & $335(43,8 \%)$ & $488(39,7 \%)$ \\
\hline DLQI-Score (0-30) $\dagger$ & $11,5(10,0)$ & $12,3(12,0)$
\end{tabular}

DLQI-Score (0-30)† 11,5 $(10,0)$

$\dagger$ Die Werte sind als Mittelwerte (Median) angegeben. 

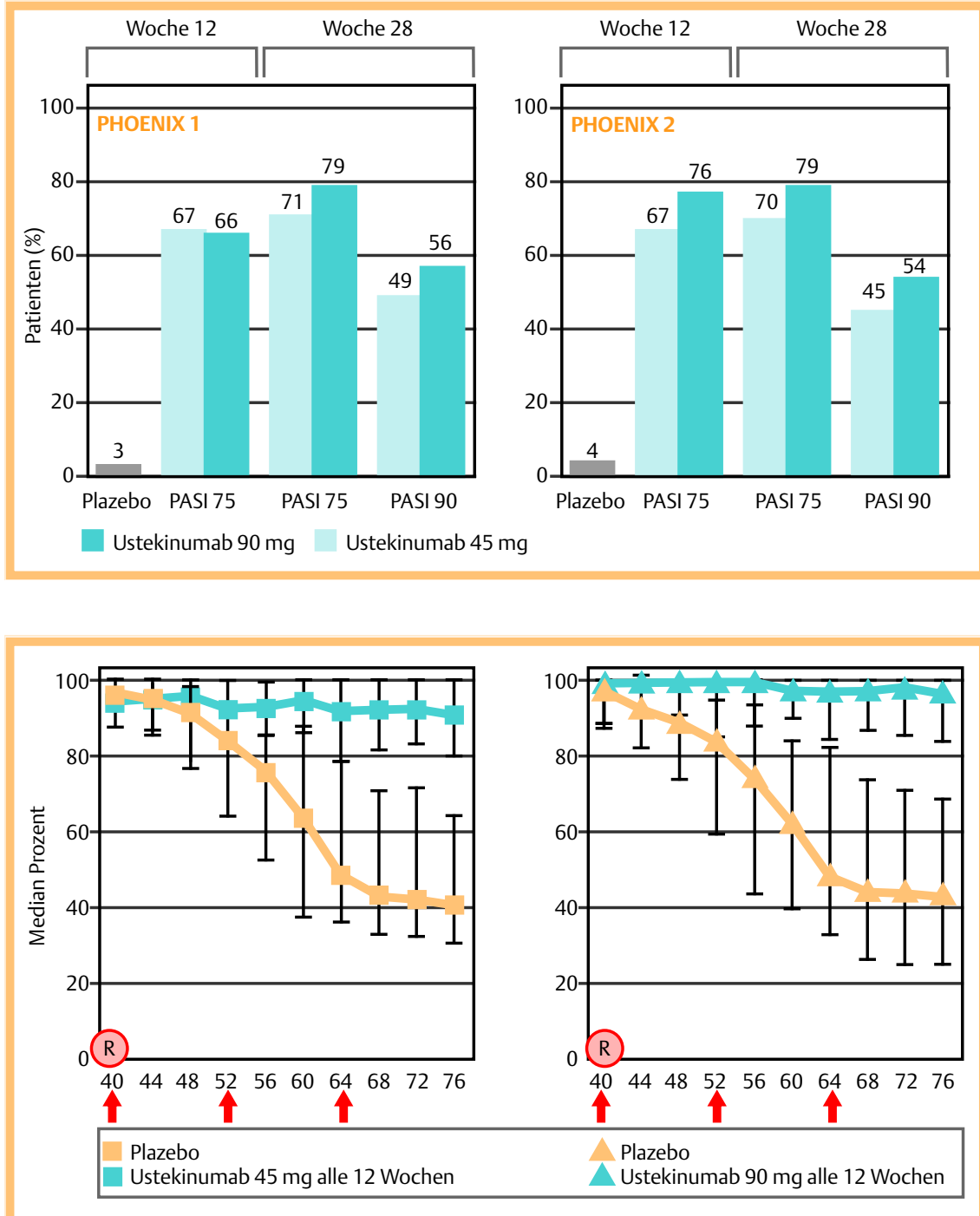

Abb. 1 Ansprechraten PASI 75 und PASI 90 zu Woche 12 und Woche 28 unter Ustekinumab $45 \mathrm{mg}$ und $90 \mathrm{mg}$ und Plazebo; primärer Endpunkt war der Anteil der Patienten mit PASI 75 zu Woche 12. schwere Psoriasis; die mittlere Erkrankungsdauer betrug 20 Jahren, im Mittel waren mehr als ein Viertel der Körperoberfläche betroffen, der mittlere PASI betrug etwa 20. Im Durchschnitt waren die Patienten sehr stark durch die Psoriasis beeinträchtigt, der mittlere DLQI lag bei 12. Eine Psoriasis-Arthritis hatten in PHOENIX 1 ein Drittel und in PHOENIX 2 etwa ein Viertel der Studienteilnehmer. Bei fast allen Patienten war eine topische Therapie erfolgt, bei zwei Drittel der Patienten eine Phototherapie und bei der Hälfte eine konventionelle systemische oder biologische Therapie (Daten nicht gezeigt).

\section{Wirksamkeit \\ $\nabla$}

In beiden Studien war Ustekinumab in beiden Dosierungen einer Behandlung mit Plazebo in der Wirksamkeit deutlich überlegen. Den primären Endpunkt - PASI 75 in Woche 12 mit Injektionen in Woche 0 und 4 - erreichten in PHOENIX $167,1 \%$ der mit $45 \mathrm{mg}$ Ustekinumab behandelten Patienten und 66,4\% der mit $90 \mathrm{mg}$ Ustekinumab behandelten Patienten, aber nur 3,1\% der Patienten in der Plazebogruppe (jeweils $\mathrm{p}<0,0001$ vs. Plazebo). In PHOENIX 2 lag der Anteil der Patienten mit PASI-75-Antwort bei $66,7 \%$ (45 mg Ustekinumab) bzw. 75,7\% (90 mg Ustekinu- mab) im Vergleich zu 3,7\% bei mit Plazebo behandelten Patienten ( $\mathrm{p}<0,0001$ vs. Plazebo) ( $\bullet$ Abb. 1).

In Woche 28 - d. h. nach Injektionen an Woche 0, 4 und 16 - erhöhte sich der Anteil der Patienten mit PASI-75-Antwort weiter auf $71 \%$ bzw. $79 \%$ in PHOENIX 1 und $70 \%$ bzw. $79 \%$ in PHOENIX 2 (45 mg bzw. 90 mg Ustekinumab; Abb.1). Die Besserung des PASI trat rasch ein: In den Verum-Gruppen hatten bereits zu Woche 2 signifikant mehr Patienten einen PASI 50 und zu Woche 4 einen PASI 75 erreicht als in den Plazebo-Gruppen (zu Woche 4 jeweils $\mathrm{p}<0,0001$ vs. Plazebo). Auch für eine PASI-50bzw. PASI-90-Antwort war der Anteil der Patienten in den Verum-Gruppen nach 12 Wochen und 28 Wochen signifikant höher: Bei fast allen Patienten besserte sich die Psoriasis um 50\%, bei jedem zweiten Patienten sogar um 90\% ( Abb. 1), letzteres war in den Plazebo-Gruppen nur bei ca. 1-2\% der Fall. Patienten, die in den ersten 12 Wochen Plazebo erhielten, sprachen nach Umstellung auf Ustekinumab bis Woche 28 vergleichbar gut an wie die Patienten, die Ustekinumab von Anfang an erhielten.

In der PHOENIX-1-Studie zeigte sich ein guter Erhalt der Wirksamkeit bei Weiterbehandlung mit Injektionen alle 12 Wochen. Wie in Abb. 2 dargestellt, blieb eine PASI-75-Antwort bei Weiterbehandlung von Patienten, die an Woche 28 und 40 eine 


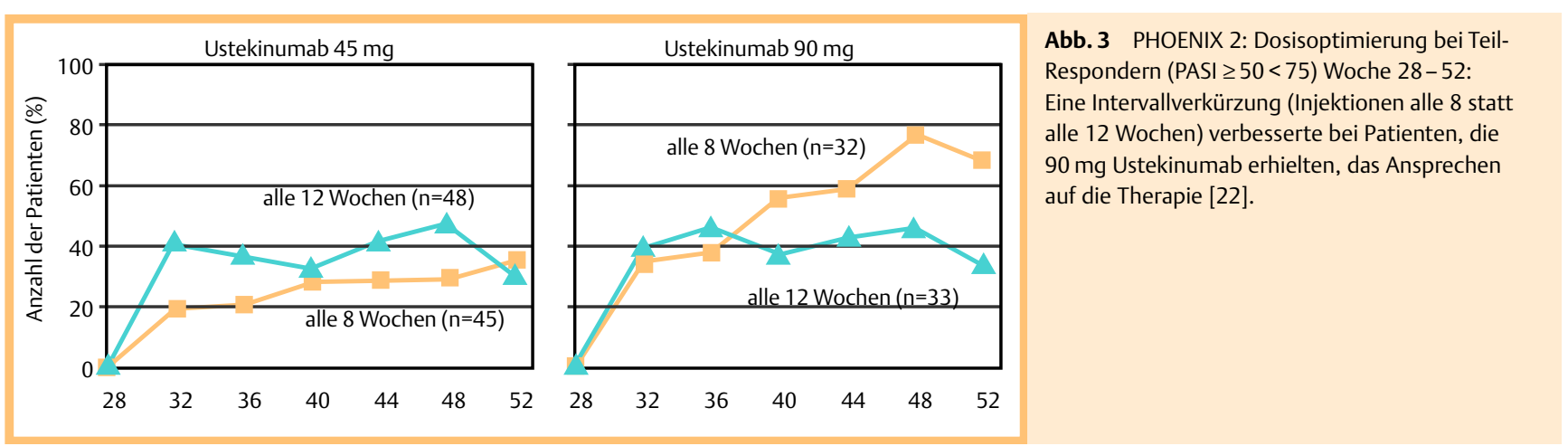

PASI-75-Antwort hatten, bis Woche 76 weitgehend erhalten (ब Abb. 2).

Bei den Patienten, die nach 40 Wochen auf Plazebo umgestellt wurden, verschlechterte sich der PASI ab Woche 44 allmählich mit einer medianen Zeit bis zum PASI-75-Verlust von 15 Wochen. Ein Rebound-Phänomen wurde nicht beobachtet. Wurde die Behandlung mit der ursprünglichen Dosis von Ustekinumab bei Patienten wieder angesetzt, wenn ein Verlust der bis Woche 40 erreichten Verbesserung des PASI von 50\% eingetreten war $(n=196)$, erreichten innerhalb von 12 Wochen $85,6 \%$ der Patienten erneut eine PASI-75-Antwort.

In der PHOENIX-2-Studie zeigte sich bei Patienten, die an Woche 28 eine PASI-75-Antwort erreicht hatten, ein Erhalt der klinischen Wirksamkeit über mindestens 1 Jahr. Auch diese Patienten werden aktuell noch weiter behandelt. Die Anzahl der Patienten die an Woche 28 in der PHOENIX-2-Studie eine mindestens $50 \%$ ige aber weniger als $75 \%$ ige Verbesserung des PASI erreicht hatten (partielles Ansprechen) betrug in der 45-mg-Gruppe $22,7 \%$ und in der $90-\mathrm{mg}$-Gruppe $15,8 \%$ der Patienten. Im Vergleich zu Patienten, die an Woche 28 eine PASI-75-Antwort erreichten $(n=589)$, unterschieden sich die Patienten, die nur partiell ansprachen $(n=158)$, vor allem durch ein höheres Körpergewicht sowie auch durch eine tendenziell stärker ausgeprägte Psoriasis mit häufigerer systemischer Vorbehandlung bzw. Nichtansprechen auf eine konventionelle systemische Therapie oder ein Biologikum in der Vorgeschichte. Zudem ließen sich bei insgesamt $12,7 \%$ der nur partiell ansprechenden Patienten im Vergleich zu 2\% bei ansprechenden Patienten (PASI-75-Antwort) an Woche 52 Antikörper gegen Ustekinumab nachweisen. Die Talspiegel von Ustekinumab bei nur partiell ansprechenden Patienten waren um den Faktor 2 bis 3 niedriger als bei ansprechenden Patienten.

Bei Fortführung der Therapie mit Ustekinumab $45 \mathrm{mg}$ nach Woche 28 mit Injektionen alle 12 Wochen erreichten ca. 30\% der Patienten eine PASI-75-Antwort an Woche 52. Dieser Prozentsatz lag bei Verkürzung des Intervalls auf $45 \mathrm{mg}$ alle 8 Wochen nicht deutlich höher. Demgegenüber erreichten mit Injektionen von $90 \mathrm{mg}$ alle 8 Wochen ca. 70\% der an Woche 28 nur partiell ansprechenden Patienten an Woche 52 eine PASI-75-Antwort im Vergleich zu nur ca. $30 \%$ mit $90 \mathrm{mg}$ alle 12 Wochen weiter behandelten Patienten $(\bullet$ Abb. 3).

Die Intensivierung führte insgesamt zu vier- bis fünffach höheren Talspiegeln von Ustekinumab (im Mittel $0,70 \mu \mathrm{l} / \mathrm{ml}$ in der 45-mg-Gruppe und $1,45 \mu \mathrm{l} / \mathrm{ml}$ in der $90-\mathrm{mg}$-Gruppe).

\section{Lebensqualität}

$\nabla$

Die von den Patienten mithilfe des DLQI bewertete Verbesserung der Lebensqualität verhielt sich parallel zu der von den Ärzten bewerteten klinischen Verbesserung: Bei zirka $80 \%$ aller Patienten, die mit Ustekinumab behandelt wurden, besserte sich der DLQI um mindestens 5 Punkte, was als klinisch signifikante Verbesserung angesehen wird. An Woche 12 hatten gut die Hälfte der Patienten einen DLQI von 0 oder 1 erreicht, d.h sie fühlten sich durch die Psoriasis nicht (mehr) beeinträchtigt: In PHOENIX $153,1 \%$ der Patienten unter 45 mg Ustekinumab, 52,4\% unter $90 \mathrm{mg}$ Ustekinumab und 6\% unter Plazebo und in PHOENIX 2 $55,3 \%$ der Patienten unter $45 \mathrm{mg}$ Ustekinumab, 56,4\% unter 90 mg Ustekinumab und 3,2\% unter Plazebo. Der DLQI besserte sich unter Ustekinumab im Median 7 - 9 Punkte mehr als unter Plazebo. Die Besserung des DLQI hielt bei Patienten, die Ustekinumab fortlaufend erhielten, an, bei den Patienten mit Therapieunterbrechung verschlechterte sich die Lebensqualität wieder.

\section{Vertråglichkeit \\ $\nabla$}

In PHOENIX 1 und 2 hatten jeweils rund die Hälfte der Patienten in den ersten 12 Wochen mindestens 1 unerwünschtes Ereignis, ohne wesentliche Unterschiede zwischen mit Plazebo und mit Ustekinumab behandelten Patienten ( $\bullet$ Tab. 2).

Die häufigsten unerwünschten Ereignisse waren Infektion der oberen Atemwege, Nasopharyngitis, Kopfschmerzen und Gelenkschmerzen. Auch schwerwiegende unerwünschte Ereignisse oder unerwünschte Ereignisse, die zu einem Studienabbruch führten, traten etwa gleich häufig bei mit Ustekinumab und mit Plazebo behandelten Patienten auf ( $\bullet$ Tab.2). Maligne Erkrankungen wurden nicht beobachtet. Die Rate der Reaktionen an der Injektionsstelle war mit $1 \%$ in den Verum- und $0,4 \%$ in den Plazebo-Gruppen gering.

In PHOENIX 1 konnte gezeigt werden, dass das Nebenwirkungsprofil auch in der Langzeittherapie mit Plazebo vergleichbar blieb ( $\odot$ Tab. 2).

Antikörper gegen Ustekinumab entwickelten in PHOENIX 1 $5,1 \%$ und in PHOENIX $25,4 \%$ der Patienten. Die Titer waren in der Regel sehr niedrig (1/320) und eine anaphylaktische Reaktion oder eine Serum-Krankheit traten nicht auf ( $\bullet$ Tab. 3). 


\begin{tabular}{|c|c|c|c|c|}
\hline & PHOENIX 1 & & PHOENIX 2 & \\
\hline $\begin{array}{l}\text { Unerwünschte Ereignisse } \\
\text { bis Woche } 12\end{array}$ & $\begin{array}{l}\text { Placebo } \\
(n=255)\end{array}$ & $\begin{array}{l}\text { Ustekinumab* } \\
(n=510)\end{array}$ & $\begin{array}{l}\text { Plazebo } \\
(n=410)\end{array}$ & $\begin{array}{l}\text { Ustekinumab* } \\
(\mathrm{n}=\mathbf{8 2 0})\end{array}$ \\
\hline Patienten mit $\geq 1$ UE, $\mathrm{n}(\%)$ & $122(47,8 \%)$ & $277(54,3 \%)$ & $202(49,3 \%)$ & $412(50,2 \%)$ \\
\hline Patienten mit $\geq 1$ SUE, n (\%) & $2(0,8 \%)$ & $6(1,2 \%)$ & $8(2,0 \%)$ & $13(1,6 \%)$ \\
\hline Patienten mit $\geq 1$ Infektion, n (\%) & $68(26,7 \%)$ & $146(28,6 \%)$ & $82(20,0 \%)$ & $176(21,5 \%)$ \\
\hline $\begin{array}{l}\text { Patienten mit } \geq 1 \text { schweren Infektion, } \\
\mathrm{n}(\%)\end{array}$ & $1(0,4 \%)$ & $2(0,4 \%)$ & $2(0,5 \%)$ & $1(0,1 \%)$ \\
\hline $\begin{array}{l}\text { Patienten mit } \geq 1 \text { Krebserkrankung, } \\
\mathrm{n}(\%)\end{array}$ & $0(0 \%)$ & $0(0 \%)$ & $1(0,2 \%)$ & $1(0,1 \%)$ \\
\hline Patienten mit $\geq 1$ MI/Schlaganfall, $\mathrm{n}$ (\%) & $0(0 \%)$ & $0(0 \%)$ & $0(0 \%)$ & $0(0 \%)$ \\
\hline
\end{tabular}

Tab. 2 Unerwünschte Ereignisse unter der Therapie mit Ustekinumab in Phoenix 1 und Phoenix 2 im Vergleich zu Plazcebo.

* kominierte Daten der $45 \mathrm{mg}$ - und $90 \mathrm{mg}$-Gruppen

\begin{tabular}{|c|c|c|}
\hline $\begin{array}{l}\text { Unerwünschte Ereignisse während } \\
\text { Langzeittherapie (Wochen 40-76) }\end{array}$ & $\begin{array}{l}\text { Erhaltungstherapie } \\
\text { (Ustekinumab) }(n=161)\end{array}$ & $\begin{array}{l}\text { Unterbrochene Therapie } \\
\text { (Plazebo) }(n=160)\end{array}$ \\
\hline $\begin{array}{l}\text { Patienten mit einem oder mehreren } \\
\text { unerwünschten Ereignissen (UE) }\end{array}$ & $108(67,1 \%)$ & $121(75,6 \%)$ \\
\hline \multicolumn{3}{|l|}{ übliche UEs } \\
\hline - Infektionen der oberen Atemwege & $22(13,7 \%)$ & $21(13,1 \%)$ \\
\hline - Nasopharyngitis & $16(9,9 \%)$ & $17(10,6 \%)$ \\
\hline - Arthralgie & $3(1,9 \%)$ & $12(7,5 \%)$ \\
\hline - Kopfschmerzen & $6(3,7 \%)$ & $4(2,5 \%)$ \\
\hline $\begin{array}{l}\text { UEs, die zum Absetzen des Studienmedikaments } \\
\text { führten }\end{array}$ & $3(1,9 \%)$ & $3(1,9 \%)$ \\
\hline Patienten mit $\geq$ ? 1 schweren UE & $1(0,6 \%)$ & $7(4,4 \%)$ \\
\hline \multicolumn{3}{|l|}{ UEs, die besondere Beachtung verdienen } \\
\hline - Infektionen & $71(44,1 \%)$ & $76(47,5 \%)$ \\
\hline - schwere Infektionen & $0(0 \%)$ & $2(1,3 \%)$ \\
\hline - Hautkrebs & $2(1,2 \%)$ & $0(0 \%)$ \\
\hline - Nicht-Hautkrebs & $0(0 \%)$ & $1(0,6 \%)$ \\
\hline - kardiovaskuläre Ereignisse & $0(0 \%)$ & $0(0 \%)$ \\
\hline
\end{tabular}

Tab. 3 PHOENIX 1. Sicherheitsprofil bei Langzeittherapie [21].

\section{Diskussion}

$\nabla$

Die Ergebnisse dieser beiden großen Phase-III-Studien mit fast 2000 Patienten zeigen, dass die subkutane Injektion von $45 \mathrm{mg}$ oder 90 mg Ustekinumab zu einer schnellen, signifikanten Verbesserung der mittelschweren bis schweren Psoriasis führt. Zwei Drittel der Patienten mit mittelschwerer und schwerer Psoriasis sprachen an Woche 12 und nahezu drei Viertel an Woche 28 mit einer mindestens 75\%igen Verbesserung des PASI an. Die vorliegenden Daten deuten auf einen Erhalt der guten Wirksamkeit in der Langzeittherapie mit Injektionen alle 12 Wochen, wobei aktuell Daten über einen Zeitraum von 76 Wochen vorliegen. Parallel zu einer Reduktion der Hautsymptome zeigten beide Studien übereinstimmend eine deutliche Besserung der Lebensqualität. Bisher wurden noch keine direkten Vergleichsdaten zu anderen biologischen Therapien veröffentlicht, eine Vergleichsstudie von Ustekinumab gegen Etanercept wird derzeit ausgewertet. Die Daten der PHOENIX-1 und -2-Studien sind mit der Annahme vereinbar, dass Ustekinumab bei mittelschwerer bis schwerer Psoriasis eine Wirksamkeit aufweist, die der von Infusionen mit Infliximab oder Injektionen von Adalimumab vergleichbar ist $[12,13]$.

In beiden Studien zeigte sich eine gute Sicherheit und Verträglichkeit der Behandlung mit Ustekinumab. Wesentliche unerwünschte Ereignisse in den plazebokontrollierten Phasen (Woche 0 bis 12; in PHOENIX 1 auch Woche 40 bis 76) traten bei mit Ustekinumab behandelten Patienten nicht häufiger auf als bei mit Plazebo behandelten Patienten. Hier müssen weitere Er- fahrungen aus der Langzeittherapie über mehrere Jahre sowie an größeren Patientenzahlen abgewartet werden.

Die gute Wirksamkeit von Ustekinumab unterstützt das Konzept, dass Interleukin-12 und -23 eine Schlüsselrolle in der Immunpathophysiologie der Psoriasis spielen. IL-12 und IL-23 aktivieren natürliche Killerzellen und greifen in die Differenzierung der CD4-positiven naiven T-Helferzellen ein. IL-12 induziert die Differenzierung naiver T-Zellen $\mathrm{zu} \mathrm{T}_{\mathrm{H}} 1$-Zellen und fördert damit die Bildung von Zytokinen wie Interferon- $\gamma$ und Tumornekrosefaktor- $\alpha[14,15]$. IL-23 führt zur Vermehrung von $T_{H} 17-Z e l l e n$, die über die Bildung von Zytokinen wie IL-17 und IL-22 wesentlich zu den entzündlichen und epidermalen Veränderungen der Psoriasis beitragen [16-18]. Auch wenn noch nicht genau geklärt ist, wie IL-12 und IL-23 in die Pathophysiologie der Psoriasis eingreifen, scheint insbesondere IL-23 bei der Psoriasis eine Schlüsselrolle in der Entzündungskaskade zu spielen. Der Erhalt einer guten klinischen Wirksamkeit mit Injektionen von Ustekinumab nur alle 12 Wochen, bei einer terminalen Halbwertszeit von ca. 20 Tagen weist auf die Möglichkeit einer Veränderung der psoriatischen Entzündungsreaktion auch über die Antagonisierung der Zytokine IL-12 und -23 hinaus.

Während bei den meisten Patienten $45 \mathrm{mg}$ Ustekinumab zu einer guten Wirksamkeit führen, scheinen einige Patienten, vor allem solche mit einem hohen Gewicht (von $>100 \mathrm{~kg}$ ) sowie Patienten mit einem besonders schweren Verlauf der Erkrankung und unzureichendem Ansprechen auf Vortherapien von der höheren Dosis von $90 \mathrm{mg}$ zu profitieren. Noch ist nicht genau bekannt, welche Dosis in welcher Weise zur Behandlung zugelassen werden 
wird. Bisher nur als Abstrakt vorgestellte Daten einer Phase2-Studie bei Psoriasis-Arthritis zeigen, dass Ustekinumab auch bei PsA wirksam ist [19], hier müssen weitere Studien Aufschluss geben über die optimale Therapiestrategie und Dosierung.

Bei Patienten, deren Behandlung unterbrochen wurde, verschlechterten sich die Hautsymptome der Psoriasis und die Lebensqualität langsam wieder - ein Anzeichen dafür, dass bei chronisch aktiven Patienten eine kontinuierliche Therapie zum Erhalt der Wirksamkeit notwendig ist. Die Daten der PHOENIX1-Studie zeigen aber, dass eine Unterbrechung der Therapie mit Ustekinumab möglich ist und mit einem guten Ansprechen der meisten Patienten bei Wiederaufnahme der Behandlung zu rechnen ist.

Die Ergebnisse der beiden Studien legen nahe, dass Ustekinumab eine neue, hochwirksame Therapieoption der mittelschweren und schweren Psoriasis darstellt. Bei Behandlung mit 2 subkutanen Injektionen von $45 \mathrm{mg}$ im ersten Monat und nachfolgenden Injektionen alle 12 Wochen kann bei etwa drei Viertel der Patienten mit einem guten Ansprechen und Erreichen derzeit empfohlener Therapieziele [20] in der Kurz- und in der Langzeittherapie gerechnet werden. Eine Subgruppe von schweren und besonders schwer betroffenen Patienten wird wahrscheinlich besser mit der höheren Dosis von 90 mg zu behandeln sein. Derzeit werden Patienten aus beiden Studien in einer Langzeitstudie über fünf Jahre weiterbehandelt, aus der weitere Daten insbesondere auch zur Langzeitsicherheit zu erwarten sind.

\section{Abstract}

Efficacy and Safety of Ustekinumab, a Human Monoclonal Interleukin-12/-23 Monoclonal Antibody, in Patients with Psoriasis: Results from the Randomised, Double-Blind, Placebo-Controlled Phase-III-trials PHOENIX 1 and PHOENIX 2

Interleukin-12 and Interleukin-23 play important roles in the pathophysiology of psoriasis. The human monoclonal antibody ustekinumab binds to the p40 subunit which is present in both cytokines thereby blocking the binding of the cytokines to their respective receptors.

The two randomised, double-blind, placebo-controlled phaseIII-trials PHOENIX 1 and PHOENIX 2 assessed the efficacy and safety of $45 \mathrm{mg}$ and $90 \mathrm{mg}$ s.c. ustekinumab in almost 2000 patients with moderate-to-severe psoriasis.

Both studies had a placebo-controlled first part with ustekinumab injections at weeks 0 and 4 . At week $1267 \%$ and $72 \%$ of patients treated with ustekinumab 45 or $90 \mathrm{mg}$ respectively achieved an at least $75 \%$ improvement of their psoriasis area and severity index (PASI 75) compared to $4 \%$ of patients treated with placebo. The reduction of skin symptoms was associated with a significant improvement of patients' quality of life. At week $1255 \%$ and $71 \%$ of patients receiving $45 \mathrm{mg}$ and $90 \mathrm{mg}$ of ustekinumab, respectively, reported a dermatology life quality index (DLQI) of ' 0 ' or ' 1 ' consistent with a high quality of life unaffected by psoriasis (placebo $4 \%$ ). The existing data of longterm ustekinumab therapy with injections every 12 weeks document maintenance of good clinical efficacy in the majority of patients for up to 76 weeks. Ustekinumab showed a favourable safety profile and good tolerability during induction and maintenance therapy. The frequency of adverse events, serious adver- se events and adverse events leading to study termination were similar among patients treated with ustekinumab and those treated with placebo.

These data suggest that ustekinumab is an effective and safe new option for the treatment of patients with moderate-to-severe psoriasis

\section{Literatur}

1 Craig $L L$, Kimball AB, Papp KA et al. Efficacy and safety of Ustekinumab, a human interleukin-12/-23 monoclonal antibody, in patients with psoriasis: 76-week results from a randomised, double-blind, placebocontrolled trial (PHOENIX 1). Lancet 2008; 371: 1665-1674

2 Papp KA, Langley RG, Lebwohl $M$ et al. Efficacy and safety of Ustekinumab, a human interleukin-12/-23 monoclonal antibody, in patients with psoriasis: 52-week results from a randomised, double-blind, placebo-controlled trial (PHOENIX 2). Lancet 2008; 371: 1675-1684

3 Krueger JG, Bowcock AM. Psoriasis pathophysiology: current concepts of pathogenesis. Ann Rheum Dis 2005; 64 (Suppl 2): ii30 - ii36

4 Lowes MA, Bowcock AM, Krueger JG. Pathogenesis and therapy of psoriasis. Nature 2007; 445: $866-873$

5 Koo J. Population-based epidemiology study of psoriasis with emphasis on quality of life assessment. Dermatol Clin 1996; 14: 485- 496

6 Schon MP, Boehncke W-H. Psoriasis. N Engl J Med 2005; 352: 18991912

7 Krueger G, Koo J, Lebwohl M et al. The impact of psoriasis on quality of life: results of a 1998 National Psoriasis Foundation Patient-Membership Survey. Arch Dermatol 2001; 137: 280-284

8 Nickoloff BJ, Stevens SR. What have we learned in dermatology from the biologic therapies? J Am Acad Dermatol 2006; 54 (3 Suppl 2): S143-S151

9 Piskin G, Sylva-Steenland RMR, Bos JD et al. In vitro und in situ expression of IL-23 by keratocytes on healthy skin and psoriasis lesion: enhanced expression in psoriatic skin. J Immunol 2006; 176: 1908 - 1915

10 Cargill M, Schrodi SJ, Chang $M$ et al. A large-scale genetic association study confirms IL12B and leads to the identification of IL23R as psoriasis-risk genes. Am J Hum Genet 2007; 80: 273-290

11 Torti DC, Feldman SR. Interleukin-12, inerleukin-23, and psoriasis: current prospects. J Am Acad Dematol 2007; 57: 1059- 1068

12 Menter A, Tyring SK, Gordon K, et . al. Adalimumab therapy for moderate to severe psoriasis: A randomized, controlled phase III trial. J Am Acad Dermatol 2008; 58: 106-115

13 Reich $K$, Nestle FO, Papp K et al. Infliximab induction and maintenance therapy for moderate-to-severe psoriasis: a phase III, multicentre, double-blind trial. Lancet 2005; 366: 1367 - 1374

14 Trinchieri $G$. Interleukin-12 and the regulation of innate resistance and adaptive immunity. Nat Rev Immunol 2003; 3: 133-146

15 Krueger JG. The immunologic basis for the treatment of psoriasis with new biologic agents. J Am Acad Dermatol 2002; 46: 1 - 23

16 Wilson NJ, Boniface K, Chan JR et al. Development, cytokine profile and function of human interleukin 17-producing helper T cells. Nat Immunol 2007; 8: 950-957

17 Bruch-Gerharz D, Fehsel K, Suschek C et al. A proinflammatory activity of interleukin 8 in human skin: expression of the inducible nitric oxide synthase in psoriatic lesions and cultured keratinocytes. J Exp Med 1996; 184: 2007-2012

18 Richards HL, Fortune DG, Shin DB et al. Adherence to treatment in patients with psoriasis. J Eur Acad Dematol Venerol 2006; 20: 370-379

19 Gottlieb AB, Menter A, Mendelsohn A et al. Phase II randomized, placebo-controlled study of CNTO 1275, a human interleukin-12/23 monoclonal antibody, in psoriatic arthritis. Presented at: American College of Rheumatology Meeting; November 7-11, 2007; Boston, Mass. Presentation L16

20 Mrowietz U, Barth J, Boehncke WH, Reich K, Rosenbach T, Streit V, Wozel G. Therapie der Psoriasis-Arthritis und der Psoriasis vulgaris mit Infliximab. JDDG 2006; 4: 444-447

21 Leonardi C, Kimball AB, Papp K et al. Efficacy and safety of ustekinumab, a human interleukin-12/23 monoclonal antibody, in patients with psoriasis: 76-week results from a randomised, doubleblind, placebo-controlled trial (PHOENIX 1). Lancet 2008; 371: 1165-1174

22 Papp KA, Lanley RG, Lebwohl $M$ et al. Efficacy and safety of ustekinumab, a human interleukin-12/23 monoclonal antibody, in patients with psoriasis: 52-week results from a randomised, double-blind, placebo-controlled trial (PHOENIX 2). Lancet 2008; 371: 1675-1684 